\title{
BMJ Open Quality indicators for hip fracture patients: a scoping review protocol
}

\author{
Kristen B Pitzul, ${ }^{1}$ Sarah E P Munce, ${ }^{2}$ Laure Perrier, ${ }^{1}$ Lauren Beaupre,,${ }^{3,4}$ \\ Suzanne N Morin, ${ }^{5}$ Rhona McGlasson, ${ }^{6}$ Susan B Jaglal ${ }^{1,2,7}$
}

To cite: Pitzul KB,

Munce SEP, Perrier L, et al. Quality indicators for hip fracture patients: a scoping review protocol. BMJ Open 2014; 4:e06543

doi:10.1136/bmjopen-2014006543

- Prepublication history for this paper is available online. To view these files please visit the journal online (http://dx.doi.org/10.1136/ bmjopen-2014-006543).

Received 3 September 2014 Revised 26 September 2014 Accepted 6 October 2014

CrossMark

For numbered affiliations see end of article.

Correspondence to Professor Susan B Jaglal; susan.jaglal@utoronto.ca

\section{ABSTRACT}

Introduction: Hip fractures are a significant cause of morbidity and mortality and care of hip fracture patients places a heavy burden on healthcare systems due to prolonged recovery time. Measuring quality of care delivered to hip fracture patients is important to help target efforts to improve care for patients and efficiency of the health system. The purpose of this study is to synthesise the evidence surrounding quality of care indicators for patients who have sustained a hip fracture. Using a scoping review methodology, the research question that will be addressed is: "What patient, institutional, and system-level indicators are currently in use or proposed for measuring quality of care across the continuum for individuals following a hip fracture?".

Methods and analysis: We will employ the methodological frameworks used by Arksey and O'Malley and Levac et al. The synthesis will be limited to quality of care indicators for individuals who suffered low trauma hip fracture. All English peerreviewed studies published from the year 2000-most recent will be included. Literature search strategies will be developed using medical subject headings and text words related to hip fracture quality indicators and the search will be peer-reviewed. Numerous electronic databases will be searched. Two reviewers will independently screen titles and abstracts for inclusion, followed by screening of the full text of potentially relevant articles to determine final inclusion. Abstracted data will include study characteristics and indicator definitions.

Dissemination: To improve quality of care for patients and create a more efficient healthcare system, mechanisms for the measurement of quality of care are required. The implementation of quality of care indicators enables stakeholders to target areas for improvement in service delivery. Knowledge translation activities will occur throughout the review with dissemination of the project goals and findings to local, national, and international stakeholders.

\section{INTRODUCTION}

In 2000, there were approximately 1.6 million hip fractures worldwide. ${ }^{1}$ By 2050, this number is projected to increase to 6.26 million primarily due to the aging

\section{Strengths and limitations of this study}

- Guided by validated methodological frameworks, has a peer-reviewed search strategy, and follows a systematic approach to data analysis.

- This review will include quality of care indicators for hip fracture patients across the entire continuum of care, and not just within the acute care setting.

- Validated and potential quality of care indicators for hip fracture patients will be included, which will provide insight into which potential indicators require further validation research.

- The review will be limited to English language studies only.

- The quality of the evidence will not be evaluated (as this is a scoping, not systematic, review).

population..$^{2-5}$ Hip fractures are a significant cause of increased morbidity and mortality, and are most prevalent in elderly women with low bone strength. ${ }^{16-12}$ These hip fractures, also known as fragility fractures, are most often associated with osteoporosis, clinically defined by low bone mineral density. ${ }^{12}$ Fragility hip fractures occur spontaneously or from minimal trauma (eg, a fall from standing height or less) ${ }^{13}$

The high prevalence of hip fractures is concerning because hip fractures are both a cause and consequence of increased frailty: Persons who sustain a hip fracture are significantly frailer, meaning they have an increased vulnerability to adverse outcomes compared to their age-matched peers. ${ }^{11} 1415$ Evidence suggests that the extensive morbidity resulting from these hip fractures results in $30-50 \%$ of hip fracture patients not returning to their premorbid function, even 1-2 years postfracture. ${ }^{16-18}$ Furthermore, a 2010 meta-analysis concluded that the all-cause mortality risk for older adults is fivefold to eightfold higher 3 months post-hip fracture compared to agematched controls. ${ }^{19}$

Personal and societal costs of hip fractures are high. Stukel et $a l^{20}$ determined that approximately $14 \%$ of hip fracture patients 
are either readmitted to acute care or die within 30 days postdischarge from the initial acute care admission. Readmissions to hospital and subsequent fractures after a hip fracture have serious consequences for both patients (eg, increased morbidity) and the healthcare system (increased utilisation). ${ }^{21}{ }^{22}$ Furthermore, postacute healthcare utilisation in hip fracture patients is also substantial as these persons require rehabilitation. ${ }^{23} 24$

In an effort to improve quality of care for patients and create a more efficient healthcare system, mechanisms for the measurement of quality of care should be in place. Quality of care indicators are a widely accepted performance measure used to determine the deviation in actual performance from ideal performance (ie, actual care delivery vs best practice care delivery)..$^{25} 26$ The implementation of quality of care indicators enables stakeholders to target areas for improvement in service delivery to improve patient outcomes and ultimately save costs. $^{27} 28$ Examples of positive change resulting from the implementation of quality of care indicator(s) include hip fracture quality of care indicators in the UK and the WHO's surgical safety checklist. ${ }^{29}{ }^{30}$ The development of quality of care indicators may occur from a deductive approach (ie, indicators are derived from scientific evidence, followed by expert opinion if required) or an inductive approach (ie, existing quality of care data is used to develop indicators). ${ }^{31}$ Although there is no gold standard to guide quality of care indicator development, Stelfox and Straus ${ }^{32}$ suggest the approach depends on the strength of evidence for a given indicator as well as its potential impact on patient health. ${ }^{33}$

A national preconsensus meeting was held in June 2013 to garner experts' opinions on possible (ie, feasible) quality of care indicators for hip fracture patients (ie, inductive approach). However, experts felt their suggested indicators were insufficient to appropriately measure the quality of care delivery, particularly across the entire continuum of care. More information with respect to the strength and breadth of scientific evidence, particularly for potential quality of care indicators was requested. Therefore, the purpose of this study is to synthesise the evidence surrounding current quality of care indicators for patients following a hip fracture. Using a scoping review methodology, the specific research question to be addressed is: "What patient, institutional, and system-level indicators are currently in use or proposed for measuring quality of care across the continuum for individuals following a hip fracture?"

\section{METHODS AND ANALYSIS}

We will employ the methodological frameworks used by Arksey and $\mathrm{O}^{\prime}$ Malley ${ }^{34}$ as well as Levac $e t a l^{35}$ for the current scoping review. The research team has expertise in the content area and methodological approach. For the purpose of this review, quality of care indicators are defined as validated quality of care measures and potential quality of care measures that have a descriptive statement. ${ }^{28}$ Care continuum includes any interaction with the healthcare system from the acute-care to postacute-care period.

\section{Eligibility criteria}

This synthesis is limited to quality of care indicators for individuals with hip fracture caused by low trauma (eg, a fall from standing height or less). Indicators for individuals with hip fractures caused by high levels of trauma (ie, motor vehicle collisions) malignant neoplasms, and Paget's disease will be excluded. These exclusion criteria will be applied as these patients are considered to be a different population and will have different care pathways and require different measures of quality.

All study designs will be included (eg, observational studies, randomised controlled trials and qualitative studies). Quality indicators targeted at patients, institutions or the healthcare system for hip fracture care will be included. Indicators developed or proposed for either the acute care and postacute care setting will be included. Only studies or abstracts published from the year 2000-onwards, or in English will be included to ensure relevance to the current healthcare context and feasibility. Limiting the search to English language only may result in bias in results towards English language speaking countries.

\section{Search strategy and information sources}

Literature search strategies will be developed using medical subject headings (MeSH) and text words related to hip fracture quality indicators. Studies will be identified by searching Medline (OVID interface), CINAHL (EBSCO interface), EMBASE (OVID interface), AgeLine (EBSCO interface), Cochrane Central Register Controlled Trials (Cochrane Library) and PEDro (physiotherapy evidence database). A hand search of the reference lists from reviews and selected articles from Osteoporosis International, a highly relevant journal, will be made to ensure literature saturation. Finally, experts in the field of osteoporosis and hip fracture will be contacted and consulted in order to ensure that all relevant data is obtained. An experienced information specialist (LP) will conduct all of the literature searches. The search strategy will be peer reviewed by another information specialist using the Peer Review of Electronic Search Strategies (PRESS). ${ }^{36}$ This will be done to clarify the boundaries of the questions and to identify other key search terms.

\section{Study selection}

To increase the reliability of screening by the two reviewers, a pilot test of the level 1 screening form based on the eligibility criteria described above will be performed on a random sample of 50 articles. The $\kappa$ statistic will then be calculated to determine the inter-rater agreement for study inclusion. ${ }^{37}$ If necessary, the inclusion and exclusion criteria will be clarified to promote the consistent application of the selection criteria. Two 
reviewers will independently screen the titles and abstracts identified by the literature search for inclusion using the screening form (level 1 screening). The full text of potentially relevant articles will then be obtained and screened to determine final inclusion (level 2 screening). A pilot test of the level 2 screening form will be performed on approximately $1 \%$ of the articles and the inter-rater agreement for study inclusion will also be calculated. ${ }^{37}$ The involvement of a third reviewer who is knowledgeable in the research area will be available to resolve discrepancies. Studies excluded during the screening phases will be recorded along with the reason (s) for exclusion.

\section{Data items and data collection process}

Abstracted data will include study characteristics (eg, year of publication, country of study), indicator definitions (eg, length of stay defined as the number of total days stayed at institution without interruption), numerator and denominator definitions when applicable (eg, per 1000 hip fractures). The main focus of the studies will be categorised or 'charted' by target of interest (either individual, institution, or system-level) as well as the indicator's place within the continuum of care (eg, acute care setting). We will examine the purpose and components of the indicators as well as the reported measurement properties if available/applicable (eg, sensitivity and specificity). Further categories may be identified through the completion of the search and via discussion with the study team. As in the study selection process, a data abstraction form will be pilot tested, standardised, and modified if poor agreement is observed. For example, any wording on the form that may be contributing to poor agreement will be reviewed and modified as necessary. Two reviewers will independently abstract all of the data, and a discussion or the involvement or a third reviewer will resolve discrepancies. Study quality will not be assessed during the scoping review as the objective of the review is to identify gaps in the literature and highlight future areas for systematic review. ${ }^{34} 35$ This means that results from poor quality studies may be inaccurate and therefore have the potential to bias study findings.

\section{Synthesis}

The results of this scoping review will be summarised quantitatively using numerical counts and qualitatively using thematic analysis (ie, using a qualitative descriptive approach). Specifically, the results of this review will determine what individual, institutional and system-level quality of care indicators are currently in use for individuals with hip fractures. Owing to the anticipated breadth of evidence that will arise from this scoping review, there is a likelihood that a given quality of care indicator, or potential quality of care indicator, is measured in a number of different ways, is context-dependent, and its applicability may change over the study time period (ie, within the past 14 years) due to changes in best practice.
The synthesis of results will ensure these differences in measurement are highlighted in order to determine potential areas of discussion among international experts (eg, discussion of why certain measures are used, and the pros and cons of each measure). Although different healthcare contexts likely require different quality of care indicators (eg, due to different funding policies), this synthesis enables discussion of the role of context, as well as any potential areas for international synergy, or at the very least international learnings (ie, informs a consensus meeting). Trends in quality of care delivery for hip fracture patients have changed over the course of the study inclusion years (ie, within the past 14 years). These changes will be discussed in brief within our synthesis; however, priority will be given to results that are most recent as they are more consistent with the current healthcare context. This review will identify gaps in the literature as well as future areas for study either via implementation studies, consensus meeting or systematic review.

\section{ETHICS AND DISSEMINATION}

Knowledge translation (KT) activities will take place at the beginning of the review and continue throughout with dissemination of the project goals to members of Bone and Joint Canada (BJC) and Osteoporosis Canada (OC) to create awareness of the project. End-of-grant KT will also occur through these agencies and their venues (eg, print and online newsletters) as well as through conventional KT mechanisms (eg, conferences and peerreviewed journals). For example, the results of the scoping review will be presented at relevant meetings locally, nationally and internationally (eg, the Technology Evaluation in the Elderly Network Conference, the Fragility Fracture Network's International Hip Fracture Registries group, Health Quality Ontario,) and published in a peer-reviewed journal so that results are available to the appropriate academic and clinical audiences. Lastly, partnerships with local clinical programmes and/or research initiatives (eg, Toronto Rehabilitation Institute) will be made to give timely and effective application of the research results.

This scoping review will summarise the body of evidence of established and potential quality indicators for hip fracture patients across the continuum of care, thereby summarising performance measures that can be used to determine the quality of care delivery for these patients.

\footnotetext{
Author affiliations

${ }^{1}$ Institute of Health Policy, Management, and Evaluation, University of

Toronto, Toronto, Canada

${ }^{2}$ Toronto Rehabilitation Institute, University Health Network, Toronto, Canada

${ }^{3}$ Department of Physical Therapy, University of Alberta, Edmonton, Canada

${ }^{4}$ Department of Surgery, University of Alberta, Edmonton, Canada

${ }^{5}$ Department of Medicine, McGill University, Montreal, Canada

${ }^{6}$ Bone and Joint Canada, Toronto, Canada

${ }^{7}$ Department of Physical Therapy, University of Toronto, Toronto, Canada
} 
Contributors KBP was involved in writing protocol, preliminary literature review; SEPM was involved in editing of protocol, scoping review frameworks, analysis; LP was involved in editing of protocol, search strategy; LB was involved in editing of protocol, content expert input (rehabilitation); SNM was involved in editing of protocol, content expert input (clinical); RM was involved in editing of protocol, stakeholder input (BJC); SBJ was involved in project conception, editing of protocol.

Funding This work was supported by a Technology Evaluation in the Elderly Network Knowledge Synthesis Grant \# 2013-07. KBP is supported by the Women's College Research Institute through the Enid Walker Estate. LB receives salary support from the Canadian Institute of Health Research as a New Investigator and from Alberta Innovates Health Solutions as a Population Health Investigator.

Competing interests None.

Provenance and peer review Not commissioned; externally peer reviewed.

Open Access This is an Open Access article distributed in accordance with the Creative Commons Attribution Non Commercial (CC BY-NC 4.0) license, which permits others to distribute, remix, adapt, build upon this work noncommercially, and license their derivative works on different terms, provided the original work is properly cited and the use is non-commercial. See: http:// creativecommons.org/licenses/by-nc/4.0/

\section{REFERENCES}

1. Johnell O, Kanis JA. An estimate of the worldwide prevalence of disability associated with osteoporotic fractures. Osteoporos Int 2006;17:1726-33.

2. Papadimitropoulos EA, Coyte PC, Josse RG, et al. Current and projected rates of hip fracture in Canada. CMAJ 1997;157:1357-63.

3. Cooper C, Campion G, Melton LJ III. Hip fractures in the elderly: a world-wide projection. Osteoporos Int 1992;2:285-9.

4. Schneider EL, Guralnik JM. The aging of America: impact on health care costs. JAMA 1990;263:235-50.

5. Dhanwal DK, Dennison EM, Harvey NC, et al. epidemiology of hip fracture: worldwide geographic variation. Ind J Ortho 2011;45:15-22.

6. Rosell PAE, Parker MJ. Functional outcome after hip fracture: a 1-year prospective outcome study of 275 patients. Injury Int J Care Injured 2003;34:529-32.

7. Nikitovic M, Wodchis WP, Krahn MD, et al. direct health-care costs attributed to hip fractures among seniors: a matched cohort study. Osteoporos Int 2013;24:659-69.

8. Heikkinen T, Parker MJ, Jalovaara P. hip fractures in Finland and Great Britain-a comparison of patient characteristics and outcomes. Int Orthop 2001;25:349-54

9. Davidson CW, Merrilees MJ, Wilkinson TJ, et al. Hip fracture mortality and morbidity-can we do better? $N Z$ Med J 2001;114:329-32.

10. Haleem S, Lutchman L, Mayahi R, et al. Mortality following hip fracture: trends and geographical variations over the last 40years. Injury 2008;39:1157-63.

11. Magaziner J, Hawkes W, Hebel JR, et al. Recovery from hip fracture in eight areas of function. J Gerontol A Biol Sci Med Sci 2000;55: M498-507.

12. Kanis JA, Delmas $P$, Burckhardt $P$, et al. Guidelines for diagnosis and management of osteoporosis. Osteoporos Int 1997;7:390.

13. Brown JP, Josse RG; Scientific Advisory Council of the Osteoporosis Society of Canada. Clinical practice guidelines for the diagnosis and management of osteoporosis in Canada. CMAJ 2002;167:S1-34.

14. Magaziner J, Lydick E, Hawkes W, et al. Excess mortality attributable to hip fracture in white women aged 70years and older. Am J Public Health 1997;87:1630-6.
15. McMillan GJ, Hubbard RE. Frailty in older inpatients: what physicians need to know. QJM 2012;105:1059-65.

16. Bertram M, Norman $R$, Kemp L, et al. Review of the long-term disability associated with hip fractures. Inj Prev 2011;17:365-70.

17. Magaziner J, Fredman L, Hawkes W, et al. Changes in functional status attributable to hip fracture: a comparison of hip fracture patients to community-dwelling aged. Am J Epidemiol 2003;157:1023-31.

18. Norton R, Butler M, Robinson E, et al. Declines in physical functioning attributable to hip fracture among older people: a follow-up study of case control participants. Disabil Rehabil 2000;22:345-51.

19. Haentjens P, Magaziner J, Colon-Emeric CS, et al. Excess mortality after hip fracture among older women and men. Ann Intern Med 2010;152:380-90.

20. Stukel TA, Fisher ES, Alter DA, et al. association of hospital spending intensity with mortality and readmission rates in Ontario hospitals. JAMA 2012;10:1037-45.

21. Boockvar KS, Halm EA, Litke A, et al. Hospital readmissions after hospital discharge for hip fracture: surgical and nonsurgical causes and effect on outcomes. J Am Geriatr Soc 2003;51:399-403.

22. Egan M, Jaglal S, Byrne $\mathrm{K}$, et al. Factors associated with a second hip fracture: a systematic review. Clin Rehabil 2008;22:272-82.

23. Dai K, Zhang W, Fan T, et al. Estimation of resource utilization associated with osteoporotic hip fracture and level of post-acute care in China. Curr Med Res Opin 2007;23:2937-43.

24. Pasco JA, Sanders KM, Hoekstra FM, et al. The human cost of fracture. Osteoporos Int 2005;16:2046-52.

25. Agency for Healthcare Research and Quality. AHRQ quality indicators. U.S. Department of Health and Human Services. http:// www.qualityindicators.ahrq.gov/ (accessed 23 Sep 2014).

26. Berg K, Fries B, Jones R, et al. Identification and evaluation of existing quality indicators that are appropriate for use in long-term care settings. Centers for Medicare and Medicaid Sedrvices, Office Clinical Standards and Quality, 2001. Contract No: 500-95-0062/T.O.\#4.

27. Gunasekera N, Boulton C, Morris C, et al. Hip fracture audit: the Nottingham experience. Osteoporos Int 2010;21:S647-653.

28. Stelfox HT, Bobranska-Artiuch B, Nathens A, et al. Quality indicators for evaluating trauma care: a scoping review. Arch Surg 2010;145:286-95.

29. Haynes AB, Weiser TG, Berry WR, et al. A surgical safety checklist to reduce morbidity and mortality in a global population. $N$ Eng $J$ Med 2009;360:491-9.

30. Royal College of Physicians and the Falls and Fragility Fracture Audit Programme. National Hip Fracture Database (NHFD) extended report. http://www.hqip.org.uk/assets/NCAPOP-Library/ NCAPOP-2014-15/NHFD-extended-report-2014-FINAL.pdf (accessed 23 Sep 2014)

31. Stelfox HT, Straus SE. Measuring quality of care: considering conceptual approaches to quality indicator development and evaluation. J Clin Epidemiol 2013:66:1328-37.

32. Stelfox HT, Straus SE. Letter reply to Kris Doggen et al. The right indicator for the job: different levels of rigor may be appropriate for the development of quality indicators. J Clin Epidemiol 2014:67:964-5.

33. Doggen K, Lavens A, Van Casteren VV. The right indicator for the job: different levels of rigor may be appropriate for the development of quality indicator. Comment on Stelfox and Strau. J Clin Epidemiol 2014;67:963-4.

34. Arksey $\mathrm{H}$, O'Malley L. Scoping studies: towards a methodological framework. Int J Soc Res Methodol 2005;8:19-32.

35. Levac DC, Colquhoun H, O'Brien KK. Scoping studies: advancing the methodology. Implement Sci 2010;5:69.

36. Sampson M, McGowan J, Cogo E, et al. An evidence-based practice guideline for the peer review of electronic search strategies. J Clin Epidemiol 2009;62:944-52.

37. Landis JR, Koch GG. The measurement of observer agreement for categorical data. Biometrics 1977;33:159-74. 